\title{
Dengue virus causes changes of MicroRNA- genes regulatory network revealing potential targets for antiviral drugs
}

\author{
Mohamed Shahen ${ }^{1,2,3 \dagger}$, Zihu Guo ${ }^{1,2 \dagger}$, Akhtar Hussain Shar ${ }^{1,2 \dagger}$, Reham Ebaid ${ }^{4}$, Qin Tao ${ }^{1,2}$, Wenjuan Zhang ${ }^{1,2}$, \\ Ziyin Wu ${ }^{1,2}$, Yaofei Bai ${ }^{1,2}$, Yingxue Fu ${ }^{1,2}$, Chunli Zheng ${ }^{1,2}$, He Wang ${ }^{1}$, Piar Ali Shar ${ }^{1,2}$, Jianling Liu, \\ Zhenzhong Wang ${ }^{6}$, Wei Xiao ${ }^{6^{*}}$ and Yonghua Wang ${ }^{1,2^{*}}$
}

\begin{abstract}
Background: Dengue virus (DENV) is an increasing global health threat and associated with induction of both a long-lived protective immune response and immune-suppression. So far, the potency of treatment of DENV via antiviral drugs is still under investigation. Recently, increasing evidences suggest the potential role of microRNAs (miRNAs) in regulating DENV. The present study focused on the function of miRNAs in innate insusceptible reactions and organization of various types of immune cells and inflammatory responses for DENV. Three drugs were tested including antiviral herbal medicine ReDuNing (RDN), Loratadine (LRD) and Acetaminophen.

Results: By the microarray expression of miRNAs in 165 Patients. Results showed that 89 active miRNAs interacted with 499 potential target genes, during antiviral treatment throughout the critical stage of DENV. Interestingly, reduction of the illness threats using RDN combined with LRD treatment showed better results than Acetaminophen alone. The inhibitions of DENV was confirmed by decrease concentrations of cytokines and interleukin parameters; like TNF- $\alpha$, IFN- $\gamma$, TGF- $\beta 1$, IL-4, IL-6, IL-12, and IL-17; after treatment and some coagulants factors increased.

Conclusions: This study showed a preliminary support to suggest that the herbal medicine RDN combined with LRD can reduce both susceptibility and the severity of DENV.
\end{abstract}

Keywords: Dengue virus, Immunity, miRNAs expression, Microarray, Mosquito

\section{Background}

Dengue fever disease (DF)is a mosquito-borne disease caused by Dengue virus (DENV) which has prominence in the past 20 years with an expanded geographic distribution of both the viruses and the mosquitoes [1]. Its spread rate was estimated as 390 million infections annually worldwide, and it is most prevalent in the developing countries [2]. DF is one of the mosquitoborne diseases which causes death all round equatorial and sub equatorial countries. The propagation of several

\footnotetext{
* Correspondence: kanionlunwen@163.com; yh_wang@nwsuaf.edu.cn ${ }^{\dagger}$ Equal contributors

${ }^{6}$ State Key Laboratory of New-tech for Chinese Medicine Pharmaceutical Process, Lianyungang, Jiangsu 222001, China

${ }^{1}$ College of Life Science, Northwest A \& F University, Yangling, Shaanxi 712100, China

Full list of author information is available at the end of the article
}

life-threatening pathogens in human is caused by DF disease [3]. DF is caused by Dengue virus (DENV), a mosquito-borne flavivirus. DENV is a single positivestrand RNA virus of the family Flaviviridae, genus Flavivirus. Its innate immune system towards a variety of diseases in humans [4].

The symptoms of DF patients suffering dengue infection commonly exist during three to 7 days of hard flulike sickness inclusive rise fever, nausea, puke, headache and body soreness [5]. After that viral infection innate and adaptive immune response are essential for the survival of the host infected by the DENV, the virus infection induce the immune system to generate type I IFN and proinflammatory cytokines parameters [6]. The host cell starts to defend against viral invasion through an appropriate innate immune response. Response to 
viral infection must be tightly regulated to defend rapidly against it, while minimizing inflammatory damage, that leads to cellular resistance to viral infection [7].

Several clinical trials were conducted to treat DENV and reduce the impact and symptoms, but until now no any licensed medicine could deal with DF. However, supportive care with a sedative, the replacement of fluids and bed rest can be useful in some cases $[5,8]$ recommended that a dosage of Acetaminophen $1 \mathrm{~g}$ every $8 \mathrm{~h}$ for 3 days dealing with DENV might be useful, while Loratadine (LRD) tablets is an orally effective medicine (ethyl4-(8-chloro-5,6-dihydro-11H-benzo, cyclohepta [1,2-b] pyridin-11-ylidene)-1-iperidinecarboxylate) [9], that could be used to treat antihistamine and relieve other allergy symptoms [10]. Additionnaly, ReDuNing (RDN) medicine a patented traditional antipyreticdetoxicate Chinese injection medicine, has been widely used as an anti-inflammatory and anti-infectious drug in Chinese clinics [11]. Dealing with the severity of dengue demands taking caution toward bodily fluids exchanges, while using proactive therapy for bleeding [12]. RDN medicine is composed of three herbs, Artemisiae annuae L. (genus Artemisia, Asteraceae), Gardenia jasminoides J.Ellis (genus Gardenia, Rubiaceae) and Lonicera japonica Thunb. (genus Lonicera, Caprifoliaceae) [13]. RDN injection medicine exhibits promising effects against enterovirus 71 in Vero monkey cells [14]. From our previous study, some methods were experimentally established to combat inflammation and provide a systems-based strategy to find the therapeutic mechanism of traditional Chinese medicine RDN dealing with the disease [13].

Nowadays miRNAs (miRNAs) has carried out numerous cautions and applications because several experimental studies have concluded that miRNAs could perform important roles in various biological processes including viral infection, differentiation, apoptosis, metabolism, cell proliferation, development, aging, signal transduction, as well as the development and progression of the human complex illness $[15,16]$. Furthermore, miRNAs have close associations with the development, progression, and prognosis of many human illnesses [16, 17], furthermore, miRNAs are widely identified to be correlated with physiological processes: including apoptosis, differentiation, growth and stress responses, also pathological processes: including prognosis, initiation and progression of various illnesses [18]. miRNAs are small (18-25 base long) noncoding RNAs, which were identified as vital ingredients of immune precision and other biological processes, such as development infection and inflammation [19]. Indeed miRNAs have a primary function in the early differentiation of B cells and maintaining the regulatory $\mathrm{T}$-cell lineage, additionally, they regulate the differentiation of dendritic cells and macrophages via toll-like receptors [20]. Recently,
microRNAs have caused great excitement as diagnostic and therapeutic signatures of the DF [21]. However, arguments for the possibility of a major function of microRNAs in controlling target genes of DF were increased [22]. So, our studies on joint activation and inhibition of endogenous miRNAs try to fight against dengue fever that includes effects on the initial Immune reaction.

The present study aimed to evaluate the treatment activity of Acetaminophen, RDN and LRD against DF. In addition, the way of treatment using single or mixed therapy was tested.

\section{Methods}

\section{Study enrollment and investigations}

This study was performed a randomized and investigator blinded. We evaluated outpatients referred to Centre for Tropical Diseases, Guangzhou, China during March-December 2015. It was approved by the ethical committee at Guangzhou University hospital of Traditional Chinese Medicine (Guangzhou, China). Patients diagnosed as DF were recruited from first affiliated at hospital. Patients were divided into two phases; primary phase was indicator chosen through a small group of plasma specimen of 20 patients treated with Acetaminophen or RDN injection contaminated LRD tablet and 15 healthy volunteers. The secondary phase was an open evaluation with a big group number of plasma specimen from 165 patients [average (SD) age, 38 (14.11) years] and 45 healthy persons as a control [age, 39.19 (10.16) years].

\section{Diagnostic procedures}

Tested DENV specific antibodies IgG and IgM in plasma samples were obtained from screened patients by ELISA antigen enzyme-linked immunosorbent assay (ELISA, Focus Technologies, Cypress, Ca, USA) [20]. Positive IgM cases were serologically confirmed by ELISA. Moreover, DV antigen and viral RNA were detected by a dot blot immunoassay and a dengue serotype specific reverse transcription PCR [23].

\section{Microarray}

Three days post Acetaminophen or RDN contaminated LRD or non-treated infection patients group. Plasma samples were drawn in PAXgene tubes to maintain miRNAs integrity and provide a snapshot of in vivo blood expression while minimizing time and manipulation variations [24]. Total RNA was extracted by mirVana miRNAs isolation kit (Ambion, Austin, TX, USA), and was amplified and assorted according to the Affymetrix GeneChip Total Transcript Sense Target Labeling protocol [25]. Replicate miRNAs in duplicate samples were averaged and miRNAs with intensities of $\geq 50$ in all samples from each group (DF-infected or treated groups) 
were selected for calculation of the normalization factor. Expressed data were median-normalized. Following normalization, significantly differentially expressed miRNAs were identified through Volcano Plot filtering as described by Cui and Churchill [26]. The detailed information of these differentially expressed miRNAs and correlation coefficient matrix for this data are listed in the Additional file 1 and the detailed result of volcano Plots filtering differentially expressed miRNAs are listed in the Additional file 2.

\section{Drugs and patients treatment}

ReDuNing (RDN, Batch No. 100906) produced by Jiangsu Kanion Pharmaceutical Co., Ltd., (Jiangsu, China); Loratadine (LRD, batch LRD/0909180) produced by Vasudna Pharma. Chem. Ltd., China were used in the research for their potential activities against dengue virus inhibition. Experimental patients were randomly divided into 3 groups; model control (without treatment) received saline only, positive control (treated with $1.3 \mathrm{~g}$ Acetaminophen tablets) administered orally three times daily for 3 days, and third group treated with $10 \mathrm{mg}$ LRD tablets and $20 \mathrm{ml}$ RDN injection. RDN dose conversion was according to its clinical dose. LRD and RDN were administered to patients the day after stress orally and by intravenous injection, respectively, for 3 days.

\section{Network construction and analysis}

To recognize the reaction mechanism of Acetaminophen and LRD with RDN as an herbal drug in the treatment of dengue fever, all active expression miRNAs and their targets were utilized to create interactions of miRNA and a predict gene were linked to each other if the gene was a predicted target of the miRNAs distributed in human. The potential miRNA were connected with those related target which were obtained from the PharmGKB database [27], and Therapeutic Targets Database (http://bidd.nus.edu.sg/group/bidd.htm).

In pharmaceutical systems, network consists of nodes and edges (connections between nodes), that is a mathematical, computable and quantifiable description of multiple relationships beneath the complicated biological systems [28]. To explore comprehensively the interrelationship between potential targets and miRNAs expressions which will help us to select out those specific targets genes correlated with DENV disease. In the resultant networks, miRNAs predicted targets genes and enrichment functions diseases were illustrated via nodes; while edges indicated the interactions between them.

Mi-T-P network was set up by overlaying the miRNAs-target gene network with related function pathway. Initially functional pathways defined by the KEGG database (Kyoto Encyclopedia of Genes and Genomes) (http://www.genome.jp/kegg/) [29] and then constructed by combining the predict targets and their related function pathways, the Database for Annotation, Visualization and Integrated Discovery (DAVID knowledgebase) (https://david.ncifcrf.gov/) [30] were used [30] to test for enriched DEmiRs targets function and to identify biological themes among them. For each miRNAs expressions, the DAVID web service provided a ranked list of functionally relevant annotation clusters that represent a summary of several annotation categories, including GO [30], KEGG [29] and BioCarta pathways [31]. In these networks, degree is used to characterize the connectedness of a node. The degree of a node is the number of edges associated with it. The topological properties of these networks were analyzed using the Network Analysis plugin and CentiScaPe 1.2 of Cytoscape 3.2.0, a popular bioinformatics package for biological network visualization and data integration, was used to generate all displayed networks [32].

To detect functional pathways or gene ontologies that are processing of differentially expressed miRNAs and putative targets of differentially expressed miRNAs after Dengue disease treated with Acetaminophen, RDN and LRD [19].

\section{Effect of antiviral inhibition on inflammatory immune response}

The supernatants samples were collected from patients before treatment and 3 days post treatment with Acetaminophen, LRD and RDN. Concentrations of TNF- $\alpha$, IFN- $\gamma$, TGF- $\beta 1$, IL- 4 , IL-6, IL-12, and IL-17 in culture supernatants were measured by ELISA according to manufacturer's instructions [33].

\section{Coagulations factor influences}

Quantitative assessment of coagulations factor was performed in the plasma with DENV isolates using the ELISA Enzygnost (Dade Behring, Deerfield, IL, USA) according to the manufacturer's instructions. Examination, we used plasma from untreated patients. In the case of the negative control, plasma was utilized. In parallel restrained survey in presence of hirudin (special thrombin restrained) was completed.

\section{Statistical analysis}

The analyses of miRNA expression data for microarray and miRNA-Target gene networks were described in each section. Other statistical analysis of Coagulations factor influences and inflammatory immune response data were performed using Student's t-test and data are expressed as means $\pm \mathrm{SE}$, and differences with probability level $P \leq 0.05$ were considered statistically significant. 


\section{Results}

The herbal medicine is widely used in clinical practices for thousands of years with efficacy and safety. However, there were few studies on anti-dengue with herbs. In this study, we compare single therapy of Acetaminophen with a new regimen of intermittent sequential therapy with Acetaminophen, LRD and RDN in the treatment of dengue virus disease. This randomized clinical trial was performed from March to December 2015. Patients diagnosed clinically with DF were randomized in three groups: patients in arm, not received any treatment, in single therapy arm patients received Acetaminophen $1.3 \mathrm{~g}$ tablet three times daily and in other arm, patients received sequential regimen of LRD $10 \mathrm{mg}$ tablet each day and RDN $20 \mathrm{ml}$ injection once daily. We carried out the detailed analysis for (RDN single treatment, RDN with Acetaminophen and LRD with Acetaminophen) however, the results were not satisfactory (data not shown).

\section{Human miRNAs expression network and predicted target gene response to dengue virus infection}

Figure 1a shows the miRNAs and their targets. In total, this network consists of 588 nodes (89 miRNAs and 499 targets) and 1497 edges. This indicates that many targets are hit by only one miRNA, while some targets can be adjusted by numerous ligands. In the interactions between miRNAs and targets, for those active miRNAs expression, M005 exhibited the highest number of target candidate $($ degree $=134)$, followed by M002 (degree $=$ 102), M013 (degree = 93), M017 (degree = 73) and so on. As for the candidate targets, DDX3X (DEAD-Box Helicase 3, X-Linked) showed the highest degree $($ Degree $=$ 18), with PTEN (Phosphatase and Tensin Homolog, Degree $=17$ ), RORA (RAR Related Orphan Receptor A, Degree $=15$ ), PPP3R1 (Protein Phosphatase 3 Regulatory Subunit B, Alpha, Degree $=14$ ), suggesting that DDX3X and PTEN might be potential targets for dengue disease

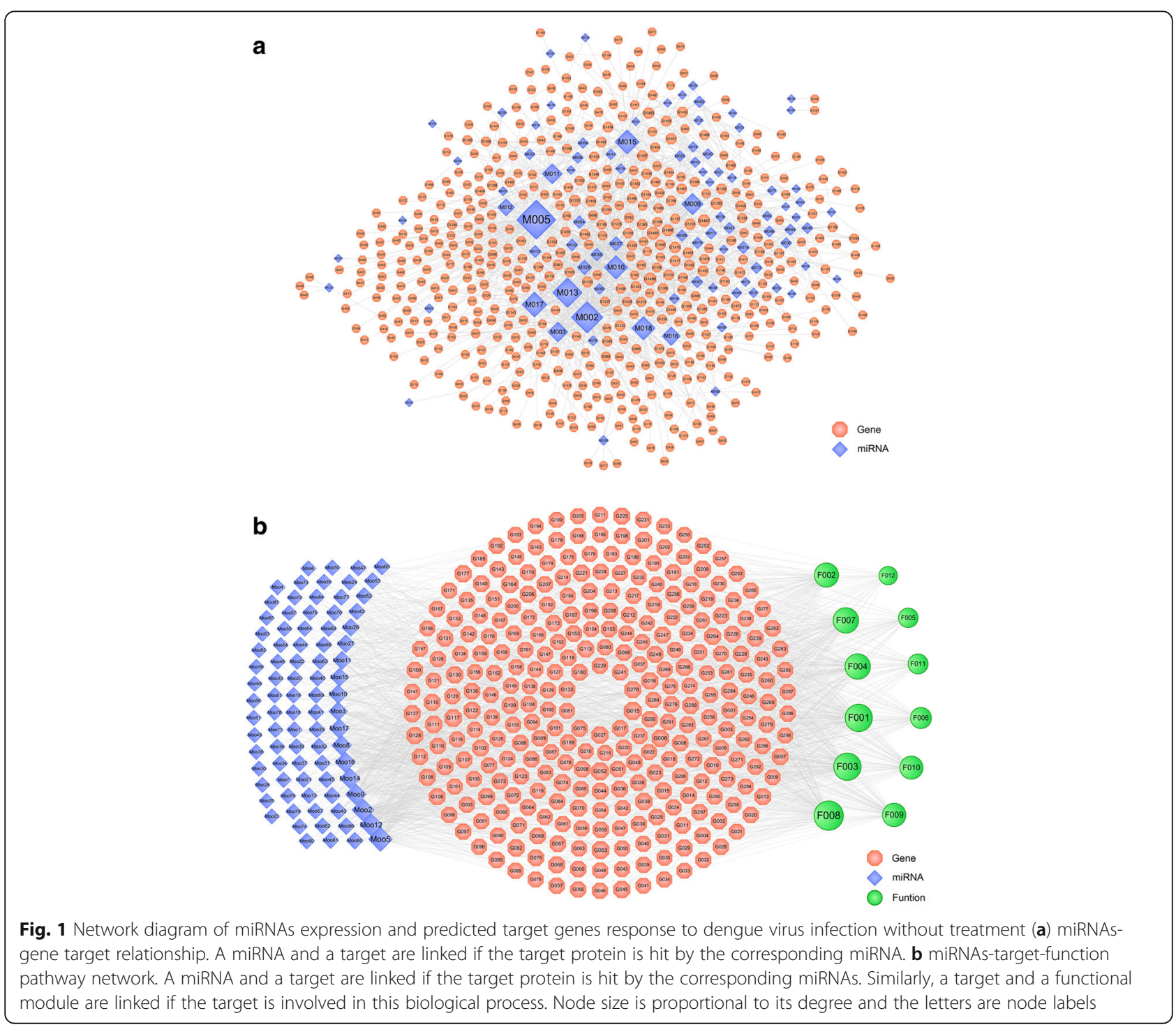


and they were modulated through related miRNAs. The detailed information of these miRNAs expressions and target genes response to DENV are listed in the Additional file 3: Table S1.

DDX3X plays an important role in the regulation of the cell proliferation, which indicated that it is involved in antiviral response against DENV infection [34], moreover DDX3X is able to stimulate the IFN promoter branches in DENV-infected cells and can be used as human immunodeficiency virus to control dengue infection. It has been suggested that the up-regulation of DDX3X may lead to treat dengue infection [35]. The suppression of PTEN gene represents a powerful strategy for ESCC therapy while PTEN has potent antiviral activity against both HIV-1 and dengue virus [36]. RORA has been implicated in several different cellular processes, including cell growth, translational control and nuclear-cytoplasmic shuttling [36]. It was previously proved that RORA played a critical role in the cellular response, such as inhibitor of NF- $\mathrm{KB}$ signaling in cell to achieve inhibitory effects on immune response programs [37, 38] Reported that RORA is a candidate transcription factor for suppressing the inflammatory response employed by $\mathrm{H} 1 \mathrm{~N} 1$ of human monocytes. PPP3R1 was reported to participate in several biological processes such as improve therapeutic strategy to treat autoimmune disorders and neurological disorders such as Alzheimer disease either responses of skeletal muscle and cardiac muscle [39].

Microarray analysis demonstrated that aberrant miRNAs expression was a remarkable characteristic in plasma after infection with DENV. Figure $1 \mathrm{~b}$ showed that Mi-T-P network consisted of 87 plasma miRNAs which were differentially down-regulated, 399 candidate genes were up-regulated and their corresponding 12 functional annotations. M005 (degree = 71) exhibited the highest active miRNAs expression, followed by M012 $($ degree $=59)$, M002 $($ degree $=58)$ and M009 $($ degree $=$ 45), indicating the multi-target properties of ingredients, which is the summary of the action mode of miRNAs expression. The targets with highest degree are listed in (Table 1). Among these targets, PTEN (Phosphatase and Tensin Homolog, degree $=21$ ) showed the highest degree, that is PTEN could interact with the most nodes in the network, which plays a key role in regulating the network as the hub target. Moreover, PTEN was followed by DDX3X (DEAD-Box Helicase 3, X-Linked, degree $=19$ ), ADRB2 (Adrenoceptor Beta 2, degree $=17$ ) and VEGFA (Vascular Endothelial Growth Factor A, degree $=16$ ) which are potential therapeutic targets genes for DENV. The detailed information of these miRNAs expressions, target genes and functional pathway are listed in the Additional file 4: Table S2. In summary, the inhibition of these six of 399 proteins
Table 1 The highest target genes response to dengue virus infection

\begin{tabular}{llll}
\hline $\begin{array}{l}\text { Gene } \\
\text { symbol }\end{array}$ & Protein name & ID & Degree \\
\hline PTEN & Phosphatase and Tensin Homolog & G015 & 21 \\
DDX3X & DEAD-Box Helicase 3, X-Linked & G052 & 19 \\
ADRB2 & Adrenoceptor Beta 2 & G053 & 17 \\
VEGFA & Vascular Endothelial Growth Factor A & G006 & 16 \\
PPP3R1 & Protein Phosphatase 3 Regulatory Subunit B, & G117 & 16 \\
& Alpha & & \\
MAP3K1 & Mitogen-Activated Protein Kinase Kinase & G164 & 15 \\
& Kinase 1 & & \\
IGF1R & Insulin Like Growth Factor 1 Receptor & G038 & 14 \\
RELA & RELA Proto-Oncogene, NF-KB Subunit & G045 & 14 \\
NRAS & Neuroblastoma RAS Viral Oncogene & G209 & 14 \\
& Homolog & & \\
CHUK & Conserved Helix-Loop-Helix Ubiquitous & G266 & 14 \\
& Kinase & & \\
MAPK1 & Mitogen-Activated Protein Kinase 1 & G016 & 13 \\
BCL10 & B-Cell CLL/Lymphoma 10 & G027 & 13 \\
ADAM10 & ADAM Metallopeptidase Domain 10 & G111 & 13 \\
RICTOR & RPTOR Independent Companion Of MTOR & G130 & 13 \\
& Complex 2 & & \\
MAPK9 & Mitogen-Activated Protein Kinase 9 & G177 & 13 \\
CCDC88A & Coiled-Coil Domain Containing 88A & G019 & 12 \\
PRKCE & Protein Kinase C Epsilon & G070 & 12 \\
\hline
\end{tabular}

(DDX3X, PTEN, RORA, PPP3R1, VEGFA, and ADRB2) may significantly decrease the replication of a DENV replicon.

In addition, there are a number of targets involve in several DENV-associated biological processes and succeed functional annotation clustering analysis were reported (Table 2). These biological processes can be summarized into several functional modules; such as regulation of cell proliferation (which is belongs to the inflammatory regulation module), regulation of programmed cell death and apoptosis.

\section{Network construction and analysis for clinical treatment}

In general, RDN Injection combined with LRD oral administration can induce the expression of miR128a, miR-389 and miR-9 to suppress dengue virus replication. [40] Previously reported that in vivo miR128a, miR-389 and miR-9 suppresses dengue virus replication by promoting NF-kB-dependent IFN production. After 3 days' post treatment with Acetaminophen, LRD and RDN, we carried out the GO enrichment and clustering analysis to find out the targets associated with the physiological features of dengue infections related to immune and inflammatory response. 
Table 2 Gene Ontology (GO) analysis of therapy target genes

\begin{tabular}{lll}
\hline Function Name & ID & Degree \\
\hline Protein kinase & F008 & 118 \\
regulation of cell proliferation & F003 & 102 \\
regulation of programmed cell death and apoptosis & F001 & 100 \\
response to cytokine stimulus & F004 & 86 \\
protein amino acid phosphorylation & F007 & 86 \\
immune response & F002 & 76 \\
protein kinase cascade & F009 & 70 \\
T cell receptor signaling pathway & F010 & 67 \\
regulation of lymphocyte activation & F006 & 49 \\
Toll-like receptor signaling pathway & F011 & 42 \\
myeloid cell differentiation & F005 & 38 \\
RIG-I-like receptor signaling pathway & F012 & 29 \\
\hline
\end{tabular}

The degree shows scores of significantly enriched 'Biological Process' categories in $\mathrm{GO}$ relative to the target genes, ( $p$ value $\leq 0.05$ )

\section{Immunity miRNAs expression profiling}

The integrative analysis of miRNAs expression recognizes a network inference of target immune response and functional interactions occurring in dengue infections patients. The result of miRNAs expression and reconstruction of a regulatory network in plasmainduced dengue symptoms post treatment by RDN with LRD are shown in Fig. 2a. The resultant Mi-T-P network which consisted of 38 differentially up-regulated expressed miRNAs and 239 integrative targets correlated with miRNAs, sorting from 2 to 64 degree and $44.4 \%$ of the genes being targeted by at least 2 miRNAs and their corresponding 12 functional annotations. The names of unique genes that are potential targets of miRNAs are PTEN (Phosphatase and Tensin Homolog) showing the highest degree (Degree $=12$ ), RELA (Proto-Oncogene, NF-KB Subunit, Degree $=12$ ), TRAF6 (TNF Receptor Associated Factor 6, Degree $=11$ ) and IL6 (Interleukin 6, Degree $=11$ ). Functional pathway analysis of anticorrelated target genes revealed various overrepresented biological processes including regulation of programmed cell death and apoptosis, regulation of cell proliferation, protein amino acid phosphorylation, immune response and response to cytokine stimulus with degree of 94, 85, 72, 57 and 55 , respectively. The information of these parameters has been listed in the Additional file 5: Table S3.

With post-treated Acetaminophen medicine as a single therapy, there is a weak correlation between miRNAs expression and their targets. (Figure 2b) shows miRNAs differential expression in the supernatant of blood samples of patients. Among the up-regulated, M003 (miRNAs, hsa-miR-200c-3p) has the highest number of target candidate $($ degree $=10)$, followed by M002 $($ degree $=6)$, M005 $($ degree $=5)$, M001 $($ degree $=2)$ and M004 (degree $=1$ ), indicating the one-target properties of ingredients. Results also showed 23 anticorrelated targets with the number of targets per miRNAs ranging from 2 to 8 ; and genes being targeted by one miRNA and their corresponding 12 functional annotations. The highest degree miRNAs are differently expressed compared with the patients treated with Acetaminophen therapy (regulation of programmed cell death and apoptosis, regulation of cell proliferation and protein amino acid phosphorylation with degree of 11,10 and 10 , respectively), which may be due to the effect of Acetaminophen on the expression of miRNAs in response to dengue virus. The detailed information of these miRNAs expressions, immune target genes and functional pathway post-treated with Acetaminophen are listed in the Additional file 6: Table S4.

Qi, et al. [41] Reported that cytokines and epigenetic regulators may be putative target genes of these miRNAs, and the pathway of biological regulation may be modulated by these miRNAs, miRNAs were classified as important ingredients of immune regulation [42]. Distinct miRNAs are capable of targeting genes that inhibit epigenetic pathways, while several miRNAs were confirmed to control down-regulation inflammatory responses by directly targeting genes encoding molecules $[43,44]$. Additionally, during DENV2 infection miRNAs were differentially expressed, among these upregulated are $\left(\mathrm{R}-4290,-4279,-625^{*}\right.$, -let-7e, $-1290,-33 \mathrm{a},-378$, $-1246,-767-5 \mathrm{p},-320 \mathrm{c},-720,-491-3 \mathrm{p},-3647,-451$ and -4286) but downregulated miRNA were (miR-106b,-20a, $-30 \mathrm{~b}$ and -3653$)$ [41].

\section{Inflammatory miRNAs expression profiling}

miRNAs expression induced dengue symptoms post treatment by RDN with LRD and (Fig. 3a) shows the interactions generated between 23 miRNAs and 39 predicted targets. The following action mode prediction showed that 15 of these miRNAs-target-pathway displayed up-regulation patterns, while the rest of them exhibited down-regulation patterns, and few of the targets are verified in previous research, such as VEGFA (Vascular Endothelial Growth Factors A) [45]. Through evaluate the molecular mechanism of RDN on MAPKs modulation, results in an activation of the mitogenactivated protein (MAP) kinase pathways, which in turn stimulates a variety of transcription factors such as NF$\mathrm{\kappa B}$ and AP-1, formerly definitely manages the production of pro-inflammatory cytokines, while downstream signaling effects that influence to inflammation [46]. Herbal medicine probably controls the infectious disease mainly based on immunomodulatory agents stimulation (such as GSK3B, MAPK14, PPAR $\gamma$ ) will probably help innate and adaptive immune, and regulation the inflammatory cytokines and 

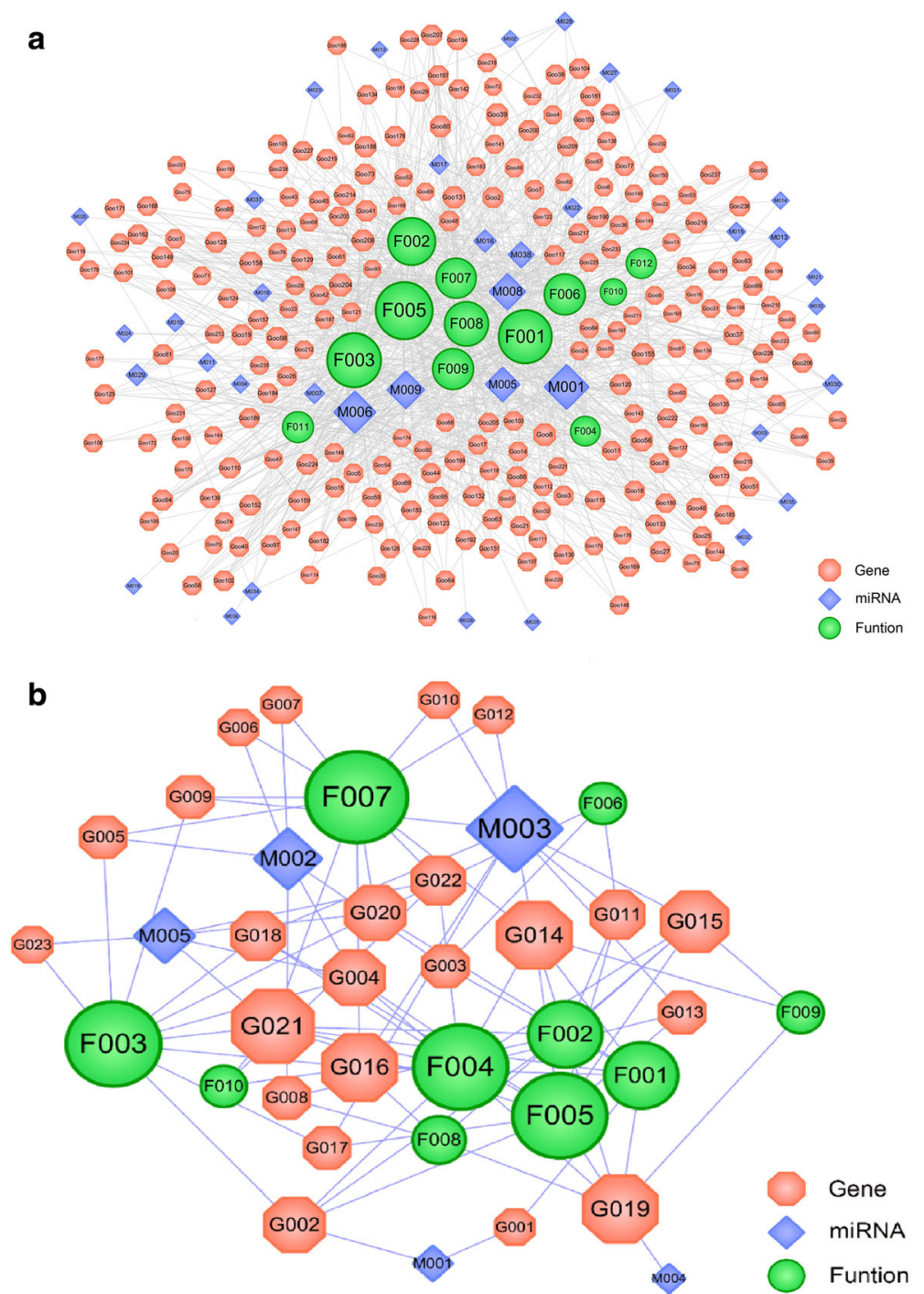

Fig. 2 Network diagram of miRNAs-immune target-function pathway response to dengue virus (a) post treated by RDN and LRD drugs. b miRNAs-immune target-function pathway, response to dengue virus post treated by Acetaminophen drug. A miRNA and a target are linked if the target protein is hit by the corresponding miRNAs. Similarly, a target and a functional module are linked if the target is involved in this biological process. Node size is proportional to its degree and the letters are node labels

proinflammatory mediators (like IL-6, IL-8, TNF- $\alpha$, COX2) [47]. More details are listed in the Additional file 7: Table S5).

In order to clarify the effect of Acetaminophen as antiinflammatory therapy and to find the relationships between the active ingredients of miRNAs and their targets, the network was generated by connecting the potential miRNAs 14 and the potential targets 24 associated with the DENV. The mean number of potential targets per potential compound was 1.045. Among the 14 potential miRNAs, 2 have high degree distributions and each of them hits more than 10 and 6 potential targets (hsa-miR-107, hsa-miR-320d). All of the 24 targets showing down-regulations were linked by 2 until 10
miRNAs and some of them are verified in previous research (Fig. 3b). The information of these parameters has been listed in the Additional file 8: Table S6.

\section{Effect of antiviral inhibition on inflammatory immune response}

LRD and RDN inhibited DV-induced proinflammatory cytokines response parameters like TNF- $\alpha$, IFN- $\gamma$, TGF$\beta 1$, IL-4, IL-6, IL-12, and IL-17 in a dose-dependent manner. That is the target of antiviral inhibition therapy to depress virus levels following treatment, toward relief symptoms of viral infection, like inflammation and haven, reducing the morbidity and the death-rate caused by the disease. Similar results were obtained in lung 


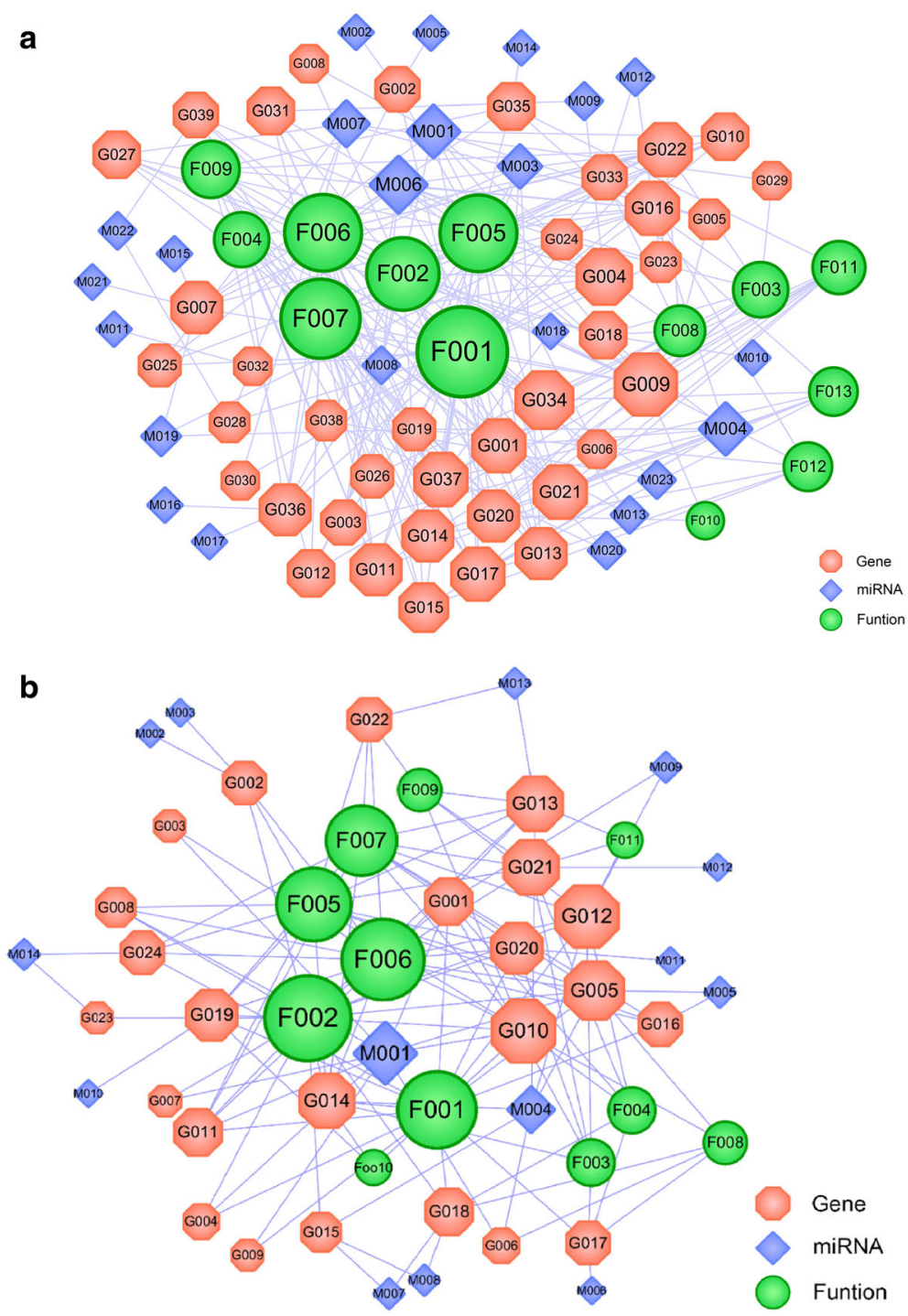

Fig. 3 Network diagram of miRNAs-inflammatory target-function pathway relationship response to dengue virus (a) post treaded by RDN and LRD drugs. $\mathbf{b}$ miRNAs- inflammatory target-function pathway, response to dengue virus post treated by Acetaminophen drug. A miRNA and a target are linked if the target protein is hit by the corresponding miRNAs. Similarly, a target and a functional module are linked if the target is involved in this biological process. Node size is proportional to its degree and the letters are node labels

injuries using RDN drugs to inhibit the levels of IFN- $\gamma$ and IL-6 [48], and RDN injection to significantly minimize the accumulations of TNF- $\alpha$ in sera and limb muscle tissues in mice [48]. Further, we examined whether the compound shown to reduce dengue immune parameters in blood were able to lower the inflammatory immune response in the patients. Cytokines parameters of all patients treated with Acetaminophen were inhibited after the treatment. Concomitant RDN with LRD, compared with Acetaminophen demonstrated greater inhibition in cytokines parameters (Fig. 4). However, cytokine concentration studies showed that concentrations of TNF- $\alpha$, IFN- $\gamma$, TGF- $\beta 1$, IL-4, IL-6, IL-12, and IL-17 were decreased in patients treated with RDN and LRD than in that treated with Acetaminophen (Fig. 4). These results indicate that the lowering of immune parameters in blood of patients by antiviral inhibition therapy may be decrease the intense inflammatory response caused by DENV and inflammatory cytokine concentrations in the patients' blood. Induction levels of several cytokines, such as IFN- $\beta$, TNF- $\alpha$, IL-6, MCP-1, and IL- $1 \beta$ in TREx-293 cells were reported to be higher upon DENV-2 infection [49]. Whereas levels of IL-6, IL-10 and IFN- $\gamma$ in mice treated with ribavirin or the combination therapy of ribavirin and RDN were all significantly decreased than the untreated group [50]. Our previous study confirmed an extremely decreased level of IL-1 $\beta$, 


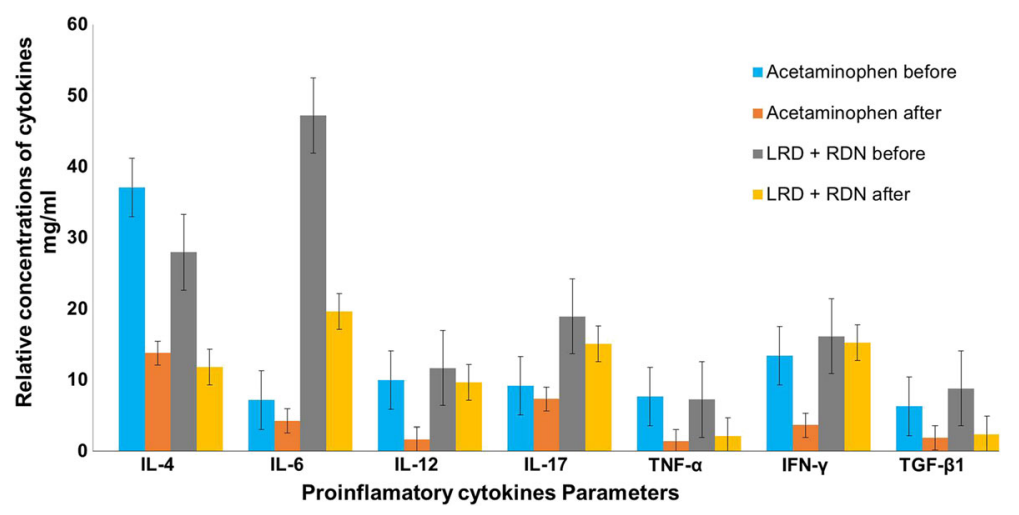

Fig. 4 Acetaminophen, LRD and RDN induces proinflammatory cytokines production. Human supernatants infected with DENV in the presence or absence of Acetaminophen, LRD and RDN at infection and persisted in the medium. The clinical scores of patients treated with various doses, $1.3 \mathrm{~g}$ tablet orally three times daily of Acetaminophen or $10 \mathrm{mg}$ of LRD tablet and $20 \mathrm{ml}$ of RDN injection one time daily. IL-4, IL-6, IL-12, IL-17, TNF-a, IFN- $\gamma$, and TGF- $\beta 1$ levels in the supernatants were measured by ELISA before treatment and $72 \mathrm{~h}$ post treatment. Data represent mean of three experiments

TNF- $\alpha$ and IL-6 parameters in RAW 264.7 cells treated with RDN and found that RDN may systematically disturb the NFL- $\mathrm{kB}$ and MAPK pathways. Therefore, RDN may block specific genes transcription partially through reducing the phosphorylation of IKB- $\alpha$ and the activation of NF-kB p65 [13].

\section{Influence of coagulation factor}

This demonstrated that not only cytokines inhibited DF, but also human coagulants factor was used as a goal to minimize viremia through dengue infection. On behalf of the above feedback, we went ahead to study the impact of Acetaminophen given orally at a dose of $1.3 \mathrm{~g}$ three time daily for 3 days. Additionally, there was another treatment group with $20 \mathrm{ml}$ RDN daily intravenous injections dose plus LRD $10 \mathrm{mg}$ tablet each day. The present study showed significant lowering of viremia whereas increasing in the all coagulants factors Coa II, Coa V, Coa VII, Coa VIII, Coa IX, Coa X and Coa XI, respectively (Fig. 5) [51]. Recommended that, there is a correlation between activation of coagulation and clinical outcome of DENV infections. Some experimental tests have recommended that DENV and immunoglobulins from patients infected with the virus can directly impact the haemostatic regularity [52].

\section{Discussion}

Treatment of DENV is presently a global infection and it is a viral disease for which there is no licensed therapeutic agent [53]. Treatment and diagnosis via new molecular-based techniques have become a new hope for early control, but are still exclusive because of their costs and normalization. DENV is the most important factors transmitted by mosquitoes worldwide and causing viral disease, almost 100 million cases of dangerous
DENV each year, resulting in severe and life-threatening [54]. Direct anti-virus treatment that reduces the risk of dengue fever can be helpful although this may need to keep all serotypes effectively [55]. Predicting potential miRNA-disease associations created valuable contributions to promoting new drugs, explaining the pathogenesis concerning disorders and medication for distinct individual complex illnesses [16]. However, we have not completed a comprehensive outlook of all miRNAs expression regulation target genes through virus-human interactions in patients of DENV. A few human miRNAs might show antiviral miRNAs. When some delusive antiviral miRNAs were blocked by locked nucleic acidmodified antisense oligoribonucleotides, the hosts miss carriage to suppress viral replication [56]. Otherwise, in few cases, human miRNAs expression might be corroborated by viruses to reshape the human intracellular medium to benefit viral replication [57].

In the present study, results showed that about 89 human miRNAs are important in the DENV interactions before medication, which are of interest in human immune and inflammatory responses. Therefore, our study provides the most human miRNAs and their predicted functions pathways mediated by them in response to virus infection. In contrast, similar studies in mammals, revealed that the miRNAs profiles were reshaped by hepatitis $C$ virus and miRNAs of human might have been correlated with the regulation of human immune systems [58]. Accordingly, virus contagion has a capability of destructing processions of cellular functions of the host on several levels, like the effects of the cell cycle or apoptosis of cells infected with viruses, including miRNAs [59]. Through cellular, miRNAs of virus-host interactions play a key regulators of gene expression [60]. Further experiments concluded 


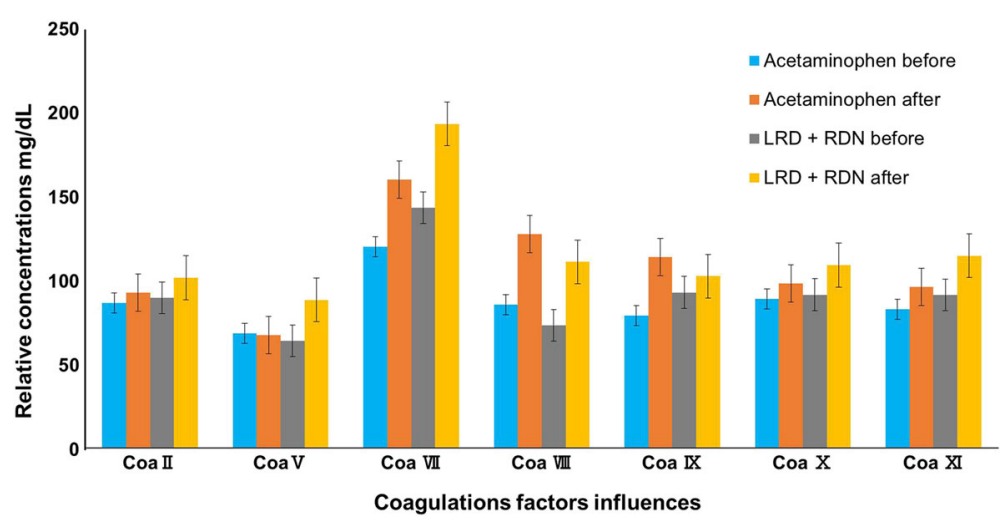

Fig. 5 Anti-viral effects of continuous treatment with Acetaminophen, LRD and RDN were used to evaluate the anti-dengue virus activities of the LRD and RDN. Patients were treated with the LRD and RDN up to 3 days. The percentages of coagulants factors Coa II, Coa V, Coa VII, Coa VIII, Coa IX, Coa X and Coa XI, increased respectively after treatment, were obtained by comparing against before treated from triplicate experiments

that the regulation of Ad6 by miR-122 could significantly improve the safety profile of the entire body rearward systemic administration. Consequently, also recognizing disease-related miRNAs could adequately improve disease biomarker detection for the medication, it is necessary to develop robust computational models to predict innovative human disease-miRNA associations [15, 61].

Microarray analysis in the present study displayed that hsa-miR-320a; hsa-miR-107 and hsa-miR-361-5p were extremely preserved in human. In addition, analyses predicted that miR-320a; one of the miRNAs highly preserved could target the Mitogen-Activated Phosphatase and Tensin Homolog (PTE), and DEAD-Box Helicase 3, $\mathrm{X}$-Linked (DDX3X). Recent studies revealed that PTEN and DDX3X were activated by invading DENV, suggesting that host might inhibit virus infection by targeting viral transcripts with host miRNAs [62]. To reduce the dengue fever impact using a potential anti-dengue medicine, we administered several known antiviral drugs (Acetaminophen, LRD and RDN), oral treatment or injection targeting DENV were found to significantly reduce viremia. Additionally, LRD combination with RDN showed a significant reduction of viremia and indicated that RDN inhibitors may be interesting drug candidates for DENV. They also have a potential for antiviral efficacy in vivo, similar to their proposed used in anti-hepatitis $\mathrm{C}$ [63], whereas Acetaminophen treatment may have little effect during dengue fever treatment.

Furthermore the potential of RDN and LRD against DENV in patient are bitten by carrier mosquitoes. The results showed that RDN could reduce the immune parameters in blood of patients if mixed with LRD tablet. The suggested mechanism was that RDN downregulated NF-B activation reducing the expression of pro-inflammatory cytokines and increasing IFITM3 expression via MAVS, giving a sign that the anti-virus effects of RDN were related to adjusting the unbalanced homeostasis of the body caused by stress response, but unrelated with direct anti-virus activity $[64,65]$ Reported that RDN could inhibit the susceptibility of influenza in restraint-stressed mice, through RDN down-regulating NF- $k B$ activation to constrict the expression of proinflammatory cytokines and enhance IFITM3 mRNA expression via MAVS. This outline suggested that the anti-influenza effects of RDN were regarding homeostasis of the body caused by stress response, however not concerning with direct anti-virus activity. RDN injection has various functions; such as absolution heat, eliminate flatus, and detoxification, clinically used in the curative of hyperpyrexia, influenza and body pain, cough, yellow sputum, and other symptoms raised by respiratory tract infection. RDN injection has been openly applied with good clinical performance [11].

Until now, it has been cloudy how dengue viremia levels contribute to the severity of the disease, after that reducing viremia by antiviral drugs to reduce the symptoms of DF [66]. Found that higher level of viremia was linked with increased DENV intensity and proposed that lowering viremia may reduce morbidity and the risk of dengue hemorrhagic fever or dengue shock syndrome. Infected mice by dengue showing increased TNF-a, IFN-g and IL-6 levels, which were recognized as triggers for DENV were also reported. We have found that reducing blood some immune parameters through the use of antiviral treatment significantly decreases proinflammatory cytokine levels and increases the concentrations of coagulants factors in the blood of treated patients. These results indicated that lowering proinflammatory cytokine parameters through timely antiviral drug treatment may improve the severity of DF symptoms and perhaps decrease the risk of progression.

Finally, this work suggests ways to prohibit the gaining of DENV which is to avoid being bitten by mosquitoes that carries the disease. Although this can be done by avoiding areas where dengue is endemic, but may be that 
is not the ideal strategy because it would require a person to avoid almost of the tropical and subtropical areas of the world, many of which are popular purpose to travel, work or at least using natural repellent for mosquitoes.

\section{Conclusions}

Based on the results of this study, it was concluded that RDN could be used as anti-dengue in vivo, to reduce the susceptibility and severity of dengue virus. This study also warrants the future in vivo anti-viral as part of the developmental process for development RDN as potential anti-dengue therapeutic. We therefore, need more investigations and specific diagnostic criteria. However, this study with a few number of sample size, gives a promising opportunity in the treatment of DENV using RDN in combination with LRD.

\section{Additional files}

Additional file 1: Differentially expressed miRNAs and Correlation coefficient matrix. (XLSX 64 kb)

Additional file 2: Volcano plots showing differentially expressed miRNAs in human supernatants infected with DENV in the presence or absence of Acetaminophen, LRD and RDN at infection and persisted in the medium. (DOCX $473 \mathrm{~kb}$ )

Additional file 3: Table S1. MiRNAs and predicted target gene response to dengue virus infection. (DOCX $61 \mathrm{~kb}$ )

Additional file 4: Table S2. MiRNAs, target gene and function pathway process response to dengue virus infection. (DOCX $56 \mathrm{~kb}$ )

Additional file 5: Table S3. MiRNAs, Immune target gene and function pathway process response to dengue virus treated by RDN with LRD. (DOCX $48 \mathrm{~kb}$ )

Additional file 6: Table S4. MiRNAs, immune target gene and function pathway process response to dengue virus treated by Acetaminophen. (DOCX 27 kb)

Additional file 7: Table S5. MiRNAs, inflammatory target gene and function pathway process response to dengue virus treated by RDN with LRD. (DOCX 29 kb)

Additional file 8: Table S6. MiRNAs, inflammatory target gene and function pathway process response to dengue virus treated by Acetaminophen. (DOCX $27 \mathrm{~kb}$ )

\section{Acknowledgements}

We are grateful to the director and the staff of the Centre for Tropical Diseases in Guangzhou University hospital in particular, the doctors and nurses of the Intensive Care Unit-for their help and support during the study.

\section{Funding}

This work was supported by Grants from Northwest A \& F University, National Natural Science Foundation of China (31170796 and 81373892). It also was supported in part by Grants from, National Natural Science Foundation of China (31540008). The research and publication fee are financially supported by the National Natural Science Foundation of China (NO. U1603285).

\section{Availability of data and materials}

All data generated or analyzed during this study are included in this published article (and its Additional files)

\section{Authors' contributions}

MS, ZG and YW conceived the project. MS, QT, WZ, AHS, ZW, YB, YF, CZ, HW and PAS performed experiments. MS and QT analyzed most data. MS, ZG, JL, ZW, WX and YW provided critical intellectual input to the study and the manuscript preparation. MS, ZG, AHS and RE wrote the manuscript. All authors read and approved the final manuscript.

\section{Ethics approval and consent to participate}

We evaluated outpatients referred to Centre for Tropical Diseases. This study was approved by the ethics committee of Guangzhou University hospital of Traditional Chinese Medicine (Guang-zhou, China).

Consent for publication

Not applicable.

\section{Competing interests}

The authors declare that they have no competing interests.

\section{Publisher's Note}

Springer Nature remains neutral with regard to jurisdictional claims in published maps and institutional affiliations.

\section{Author details \\ ${ }^{1}$ College of Life Science, Northwest A \& F University, Yangling, Shaanxi 712100, China. ${ }^{2}$ Center of Bioinformatics, Northwest A \& F University, Yangling, Shaanxi 712100, China. ${ }^{3}$ Zoology Department, Faculty of Science, Tanta University, Tanta 31527, Egypt. ${ }^{4}$ School of Environment and Safety Engineering, Jiangsu University, Jiangsu 212013, China. ${ }^{5}$ College of Life Science, Northwest University, Xi'an, Shaanxi 710069, China. ${ }^{6}$ State Key Laboratory of New-tech for Chinese Medicine Pharmaceutical Process, Lianyungang, Jiangsu 222001, China.}

Received: 10 May 2017 Accepted: 23 November 2017

Published online: 04 January 2018

\section{References}

1. Carabali M, Lim JK, Velez DC, Trujillo A, Egurrola J, Lee KS, Kaufman JS, DaSilva LJ, Velez ID, Osorio JE. Dengue virus serological prevalence and seroconversion rates in children and adults in Medellin, Colombia: implications for vaccine introduction. Int J Infect Dis. 2017; 58:27-36.

2. Behnam MA, Nitsche C, Boldescu V, Klein CD. The medicinal chemistry of dengue virus. J Med Chem. 2016; 59(12):5622-49.

3. Medlock JM, Hansford KM, Schaffner F, Versteirt V, Hendrickx G, Zeller H, Bortel WV. A review of the invasive mosquitoes in Europe: ecology, public health risks, and control options. Vector-borne Zoonotic Dis. 2012;12(6): 435-47.

4. Yu J-S, Tseng C-K, Lin C-K, Hsu Y-C, Wu Y-H, Hsieh C-L, Lee J-C. Celastrol inhibits dengue virus replication via up-regulating type I interferon and downstream interferon-stimulated responses. Antivir Res. 2017:137:49-57.

5. Pandejpong D, Saengsuri P, Rattarittamrong R, Rujipattanakul T, Chouriyagune $C$. Is excessive acetaminophen intake associated with transaminitis in adult patients with dengue fever? Intern Med J. 2015;45(6): 653-8.

6. Akira S, Uematsu S, Takeuchi O. Pathogen recognition and innate immunity. Cell. 2006:124(4):783-801.

7. Hou J, Wang P, Lin L, Liu X, Ma F, An H, Wang Z, Cao X. MicroRNA-146a feedback inhibits RIG-I-dependent type I IFN production in macrophages by targeting TRAF6, IRAK1, and IRAK2. J Immunol. 2009;183(3):2150-8.

8. Maves RC, Oré RMC, Porter KR, Kochel TJ. Immunogenicity and protective efficacy of a psoralen-inactivated dengue-1 virus vaccine candidate in Aotus Nancymaae monkeys. Vaccine. 2011;29(15):2691-6.

9. Beutler B, Eidenschenk C, Crozat K, Imler J-L, Takeuchi O, Hoffmann JA, Akira S. Genetic analysis of resistance to viral infection. Nat Rev Immunol. 2007; 7(10):753-66.

10. Patel JZ, Ahenkorah S, Vaara M, Staszewski M, Adams Y, Laitinen T, NaviaPaldanius D, Parkkari T, Savinainen JR, Walczyński K. Loratadine analogues as MAGL inhibitors. Bioorg Med Chem Lett. 2015;25(7):1436-42.

11. Wang F, Li C-y, Zheng Y-f, Li H-y, Xiao W, Peng G-p. Identification of the Allergenic Ingredients in Reduning Injection by Ultrafiltration and HighPerformance Liquid Chromatography. J Immunol Res. 2016; 2016.

12. Tang L-P, Mao Z-F, Li X-X, Chen M, Li S-B, Tsoi B, Cao L-F, Li L, Zeng J-M, Wang Z-W. ReDuNing, a patented Chinese medicine, reduces the susceptibility to H1N1 influenza of mice loaded with restraint stress. European J Integrative Med. 2014;6(6):637-45. 
13. Liu J, Sun K, Zheng C, Chen X, Zhang W, Wang Z, Shar PA, Xiao W, Wang Y. Pathway as a pharmacological target for herbal medicines: an investigation from reduning injection. PLoS One. 2015;10(4):e0123109.

14. Xu HM, Wang Y, Liu NF. Safety of an injection with a mixture of extracts from Herba Artemisiae annuae, Fructus Gardeniae and Flos Lonicerae. Pharm World Sci. 2009:31(4):458-63.

15. Chen X, Yan CC, Zhang X, You ZH, Huang YA, Yan GY. HGIMDA: heterogeneous graph inference for miRNA-disease association prediction. Oncotarget. 2016;7(40):65257-69.

16. You ZH, Huang ZA, Zhu Z, Yan GY, Li ZW, Wen Z, Chen X. PBMDA: a novel and effective path-based computational model for miRNA-disease association prediction. PLoS Comput Biol. 2017;13(3):e1005455.

17. Chen X, Liu MX, Yan GY. RWRMDA: predicting novel human microRNAdisease associations. Mol BioSyst. 2012;8(10):2792-8.

18. Pham AM, Langlois RA. Replication in cells of hematopoietic origin is necessary for dengue virus dissemination. PLoS Pathog. 2012;8(1):e1002465.

19. An JH, Ohn JH, Song JA, Yang JY, Park H, Choi HJ, Kim SW, Kim SY, Park WY, Shin CS. Changes of MicroRNA profile and MicroRNA-mRNA regulatory network in bones of Ovariectomized mice. J Bone Miner Res. 2014;29(3): $644-56$

20. Marí-Alexandre J, Sánchez-Izquierdo D, Gilabert-Estellés J, Barceló-Molina M, Braza-Boills A, Sandoval J. miRNAs regulation and its role as biomarkers in endometriosis. Int J Mol Sci. 2016:17(1):93.

21. Vlachos IS, Paraskevopoulou MD, Karagkouni D, Georgakilas G, Vergoulis T, Kanellos I, Anastasopoulos I-L, Maniou S, Karathanou K, Kalfakakou D. DIANA-TarBase v7. 0: indexing more than half a million experimentally supported miRNA: mRNA interactions. Nucleic Acids Res. 2015;43(D1):D153-9.

22. Castillo JA, Castrillón JC, Diosa-Toro M, Betancur JG, St Laurent G, Smit JM, Urcuqui-Inchima S. Complex interaction between dengue virus replication and expression of miRNA-133a. BMC Infect Dis. 2016;16(1):29.

23. De Kruif MD, Setiati TE, Mairuhu AT, Koraka P, Aberson HA, Spek CA, Osterhaus AD, Reitsma PH, Brandjes DP, Soemantri A. Differential gene expression changes in children with severe dengue virus infections. PLoS Negl Trop Dis. 2008;2(4):e215.

24. Pn L, Hammond SN, Leung JM, Kim CC, Batra S, Rocha C, Balmaseda A, Harris E. Gene expression patterns of dengue virus-infected children from nicaragua reveal a distinct signature of increased metabolism. PLoS Negl Trop Dis. 2010;4(6):e710.

25. Mollaie HR, Monavari SHR, Arabzadeh SAM, Shamsi-Shahrabadi M, Fazlalipour M, Afshar RM. RNAi and miRNA in viral infections and cancers. Asian Pac J Cancer Prev. 2013;14(12):7045-56.

26. Cui $X$, Churchill GA. Statistical tests for differential expression in CDNA microarray experiments. Genome Biol. 2003;4(4):210.

27. Klein TE, Chang JT, Cho MK, Easton KL, Fergerson R, Hewett M, Lin Z, Liu Y, Liu S, Oliver D. Integrating genotype and phenotype information: an overview of the PharmGKB project. Pharmacogenomics J. 2001;1(3):167.

28. Xu Q, Qu F, Pelkonen O. Network pharmacology and traditional Chinese medicine: INTECH Open Access Publisher; 2012.

29. Kotera M, Hirakawa M, Tokimatsu T, Goto S, Kanehisa M. The KEGG databases and tools facilitating omics analysis: latest developments involving human diseases and pharmaceuticals. Next Generation Microarray Bioinformatics: Methods Protocols. 2012:19-39.

30. Dennis G, Sherman BT, Hosack DA, Yang J, Gao W, Lane HC, Lempicki RA. DAVID: database for annotation, visualization, and integrated discovery. Genome Biol. 2003;4(9):R60.

31. Nishimura D. BioCarta. Biotech Software Internet Report: Computer Software J Scient. 2001;2(3):117-20.

32. Smoot ME, Ono K, Ruscheinski J, Wang P-L, Ideker T. Cytoscape 2.8: new features for data integration and network visualization. Bioinformatics. 2011; 27(3):431-2.

33. Jiménez VC, Bradley EJ, Willemsen AM, van Kampen AH, Baas F, Kootstra NA. Next-generation sequencing of microRNAs uncovers expression signatures in polarized macrophages. Physiol Genomics. 2014;46(3):91-103.

34. Schröder M, Baran M, Bowie AG. Viral targeting of DEAD box protein 3 reveals its role in TBK1/IKKE-mediated IRF activation. EMBO J. 2008;27(15): 2147-57.

35. Robinson LN, Tharakaraman K, Rowley KJ, Costa W, Chan KR, Wong YH, Ong LC, Tan HC, Koch T, Cain D. Structure-guided design of an anti-dengue antibody directed to a non-immunodominant epitope. Cell. 2015;162(3): 493-504.
36. Wagstaff KM, Sivakumaran $H$, Heaton SM, Harrich D, Jans DA. Ivermectin is a specific inhibitor of importin $\alpha / \beta$-mediated nuclear import able to inhibit replication of HIV-1 and dengue virus. Biochem J. 2012;443(3):851-6.

37. Zhu Y, McAvoy S, Kuhn R, Smith DI. RORA, a large common fragile site gene, is involved in cellular stress response. Oncogene. 2006;25(20):2901-8.

38. Friesenhagen J, Viemann D, Börgeling Y, Schmolke M, Spiekermann C, Kirschnek S, Ludwig S, Roth J. Highly pathogenic influenza viruses inhibit inflammatory response in Monocytes via activation of Rar-related orphan receptor RORa. J Innate Immunity. 2013;5(5):505-18.

39. Hand BD, Kostek MC, Ferrell RE, Delmonico MJ, Douglass LW, Roth SM, Hagberg JM, Hurley BF. Influence of promoter region variants of insulin-like growth factor pathway genes on the strength-training response of muscle phenotypes in older adults. J Appl Physiol. 2007;103(5):1678-87.

40. W-y J. Therapeutic wisdom in traditional Chinese medicine: a perspective from modern science. Discov Med. 2009:5(29):455-61.

41. Qi Y, Li Y, Zhang L, Huang J. microRNA expression profiling and bioinformatic analysis of dengue virus-infected peripheral blood mononuclear cells. Mol Med Rep. 2013;7(3):791-8.

42. Gracias DT, Katsikis PD. MicroRNAs: key components of immune regulation. Adv Exp Med Biol. 2011; 780:15-26

43. Sato F, Tsuchiya S, Meltzer SJ, Shimizu K. MicroRNAs and epigenetics. FEBS J. 2011;278(10):1598-609

44. Ceppi M, Pereira PM, Dunand-Sauthier I, Barras E, Reith W, Santos MA, Pierre P. MicroRNA-155 modulates the interleukin-1 signaling pathway in activated human monocyte-derived dendritic cells. Proc Natl Acad Sci. 2009;106(8): 2735-40.

45. Mangione JN, Huy NT, Lan NTP, Mbanefo EC, Ha TTN, Bao LQ, Nga CTP, Van Tuong V, Van Dat T, Thuy $T$. The association of cytokines with severe dengue in children. Tropical Med Health. 2014;42(4):137.

46. Thalhamer T, McGrath M, Harnett M. MAPKs and their relevance to arthritis and inflammation. Rheumatology. 2008:47(4):409-14.

47. Yang $H$, Zhang W, Huang C, Zhou W, Yao Y, Wang Z, Li Y, Xiao W, Wang Y. A novel systems pharmacology model for herbal medicine injection: a case using reduning injection. BMC Complement Altern Med. 2014;14(1):430.

48. Zuo L, Chen Y, Zhang W: [Reduning injection inhibits replication and proliferation of mouse cytomegalovirus and down-regulates the expressions of IFN- $\gamma$ and IL-6 in mouse cytomegalovirus pneumonia]. Xi bao yu fen zi mian yi xue za zhi= Chinese J Cellular and Molecul Immunol 2013, 29(12):1242-1244.

49. Simon-Loriere E, Lin R-J, Kalayanarooj SM, Chuansumrit A, Casademont I, Lin S-Y, Yu H-P, Lert-Itthiporn W, Chaiyaratana W, Tangthawornchaikul N. High Anti-Dengue Virus Activity of the OAS Gene Family is Associated With Increased Severity of Dengue. J Infect Dis. 2015; 212(12):2011-2020.

50. Yuexia M, Wei Z, Zhongpeng Z, Min L, Jian L, Yuguang W. Combination of ribavirin and reduning protects mice against severe pneumonia induced by H1N1 influenza a virus. J Tradit Chin Med. 2016;36(2):181-6.

51. Mairuhu A, Mac Gillavry M, Setiati T, Soemantri A, Ten Cate H, Brandjes D, van Gorp E. Is clinical outcome of dengue-virus infections influenced by coagulation and fibrinolysis? A critical review of the evidence. Lancet Infect Dis. 2003;3(1):33-41.

52. Monroy V, Ruiz B. Participation of the dengue virus in the fibrinolytic process. Virus Genes. 2000;21(3):197-208.

53. Whitehorn J, Nguyen CW, Khanh LP, Kien DTH, Quyen NTH, Tran NTT, Hang NT, Truong NT, Hue Tai LT, Cam Huong NT. Lovastatin for the treatment of adult patients with dengue: a randomized, double-blind, placebo-controlled trial. Clin Infect Dis. 2015; 62(4):468-76.

54. Chan CY, Ooi EE. Dengue: an update on treatment options. Future Microbiol. 2015;10(12):2017-31.

55. Bishop BM. Potential and emerging treatment options for Ebola virus disease. Ann Pharmacother. 2015; 49(2):196-206.

56. Li S, Duan X, Li Y, Liu B, McGilvray I, Chen L. MicroRNA-130a inhibits HCV replication by restoring the innate immune response. J Viral Hepat. 2014; 21(2):121-8

57. Lazar DC, Morris KV, Saayman SM. The emerging role of long non-coding RNAs in HIV infection. Virus Res. 2016;212:114-26.

58. Diamond MS, Pierson TC. Molecular insight into dengue virus pathogenesis and its implications for disease control. Cell. 2015;162(3):488-92.

59. Huang $T, X u D, Z$ hang $X$. Characterization of host microRNAs that respond to DNA virus infection in a crustacean. BMC Genomics. 2012;13(1):159.

60. Zhao Y, Xu H, Yao Y, Smith LP, Kgosana L, Green J, Petherbridge L, Baigent SJ, Nair V. Critical role of the virus-encoded microRNA-155 ortholog in the induction of Marek's disease lymphomas. PLoS Pathog. 2011;7(2):e1001305. 
61. Chen X, Wu QF, Yan GY. RKNNMDA: ranking-based KNN for MiRNA-disease association prediction. RNA Biol. 2017;14(7):952-62

62. Janssen HL, Reesink HW, Lawitz EJ, Zeuzem S, Rodriguez-Torres M, Patel K, van der Meer AJ, Patick AK, Chen A, Zhou Y. Treatment of HCV infection by targeting microRNA. N Engl J Med. 2013;368(18):1685-94.

63. Castro EF, Fabian LE, Caputto ME, Gagey D, Finkielsztein LM, Moltrasio GY, Moglioni AG, Campos RH, Cavallaro LV. Inhibition of bovine viral diarrhea virus RNA synthesis by thiosemicarbazone derived from 5, 6-dimethoxy-1indanone. J Virol. 2011;85(11):5436-45.

64. L-p T, Xiao W, Li Y-F, Li H-B, Wang Z-Z, Yao X-S, Kurihara H, He R-R. Antiinflammatory effects of Reduning injection (热毒宁注射液) on Lipopolysaccharide-induced acute lung injury of rats. Chin J Integr Med. 2014;20(8):591-9.

65. Kurihara H. Anti-inflammatory effects of Reduning injection (热毒宁注射液) on lipopolysaccharide-induced acute lung injury of rats. Chinese J Integrative Med. 2014;20(8):591-9.

66. Schul W, Liu W, Xu H-Y, Flamand M, Vasudevan SG. A dengue fever viremia model in mice shows reduction in viral replication and suppression of the inflammatory response after treatment with antiviral drugs. J Infect Dis. 2007:195(5):665-74.

Submit your next manuscript to BioMed Central and we will help you at every step:

- We accept pre-submission inquiries

- Our selector tool helps you to find the most relevant journal

- We provide round the clock customer support

- Convenient online submission

- Thorough peer review

- Inclusion in PubMed and all major indexing services

- Maximum visibility for your research

Submit your manuscript at www.biomedcentral.com/submit
Biomed Central 